\title{
Growth and Photoluminescence Study of ZnSe Quantum Dots
}

\author{
Y.H. CHANG, M.H. CHIENG, C.C. TSAI, M.C. HARRIS LIAO, \\ and Y.F. CHEN \\ Department of Physics, National Taiwan University, Taipei 106, Taiwan, Republic of China
}

We report detailed photoluminescence (PL) studies of ZnSe quantum dots grown by controlling the flow duration of the precursors in a metal-organic chemical vapor deposition system. The growth time of the quantum dots determines the amount of blue shift observed in the PL measurements. Blue shift as large as 320 meV was observed, and the emission was found to persist up to room temperature. It is found that changing the flow rate and the total number of quantum dot layers also affect the peak PL energy. The temperature dependence of the peak PL energy follows the Varshni relation. From analyzing the temperaturedependent integrated intensity of the photoluminescence spectra, it is found that the activation energy for the quenching of photoluminescence increases with decreasing quantum dot size, and is identified as the binding energy of the exciton in ZnSe quantum dot.

Key words: ZnSe, quantum dots, exciton, photoluminescence

\begin{abstract}
Quantum dots (QDs) have received much attention because of their potential applications in optical and opto-electronics devices.1,2 Various ways of producing QDs have been demonstrated. ${ }^{3-5}$ Self-organized QDs attracted the most attention because of their nondestructive and non-growth-interrupted nature. InAs quantum dots on $\mathrm{GaAs}^{6}$ and Ge quantum dots on $\mathrm{Si}^{7}$ are well-known examples. These quantum dots were found to grow under the Stranski-Krastanov mode: a layer was first grown in two-dimension, and threedimensional islands were formed after some critical thickness was reached. These dots were formed to relax strain energy without creating dislocations between the dots and the underlying layer, and are epitaxially and coherently grown. The fact that the QDs are defect free is an important reason why QDs usually have very good optical properties.

Recently, we showed that high quality QDs could be grown in a metal-organic chemical vapor deposition system by controlling the flow duration of the precursors. ${ }^{8}$ The basic idea of the approach is to embed QDs directly in another material with wider bandgap but without introducing a two-dimensional layer. To do so, a rough surface was first prepared as a starting layer. QDs were then grown on this rough surface. Dot size was controlled by growth duration. By using this
\end{abstract}

(Received May 14, 1999; accepted August 16, 1999) method, we were able to grow ZnSe QDs with good properties. In this paper, we report detailed photoluminescence (PL) studies of ZnSe quantum dots grown with different growth parameters. The growth time of the QDs determines the amount of blue shift of the PL from these QDs. Blue shifts ranging from $320 \mathrm{meV}$ to $190 \mathrm{meV}$ were observed for growth duration time between $1 \mathrm{~s}$ to $5 \mathrm{~s}$ and the emission persists up to room temperature. However, for growth time larger than $5 \mathrm{~s}$, only very weak defect-related emission could be detected. The temperature dependence of the peak emission energy was found to decrease with increasing temperature. Effect of the flow rate of the precursor on the blue shift was studied. We found that, with the same growth duration, the smaller the DESe flow rate of the precursor, the larger the blue shift. We also found that when there are more layers of quantum dots the PL intensity increases, the peak PL energy decreases, and the spectrum become narrower. From the temperature-dependence of the integrated PL intensity, it is found that the activation energy for the quenching of PL decreases with increasing dot size. The activation energy is identified as the binding energy of exciton in the QDs.

The samples were grown by using a homemade metal-organic chemical vapor deposition system. Dimethylzinc (DMZn), diethylselenide (DESe), and diethylsulfide (DES), were used as precursors. Typi- 


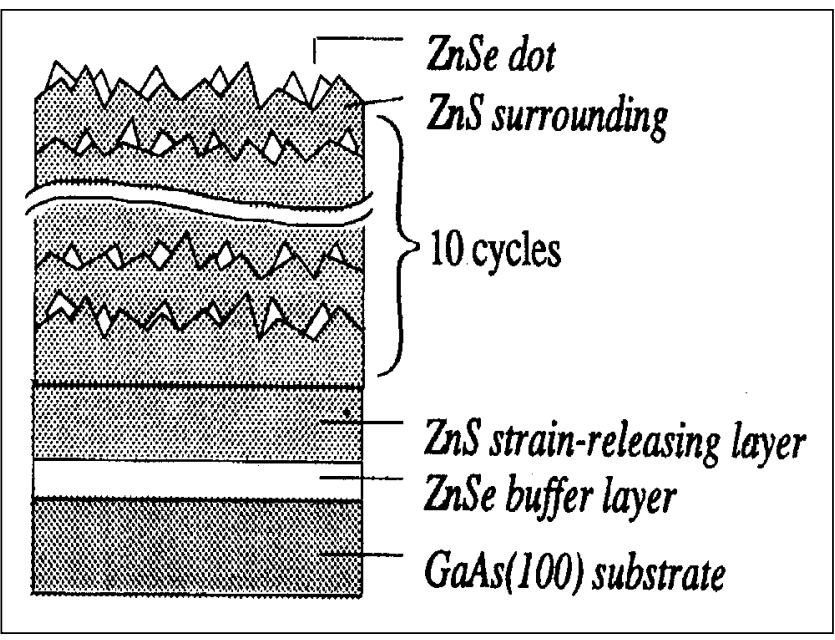

Fig. 1. Schematic diagram of sample structure.

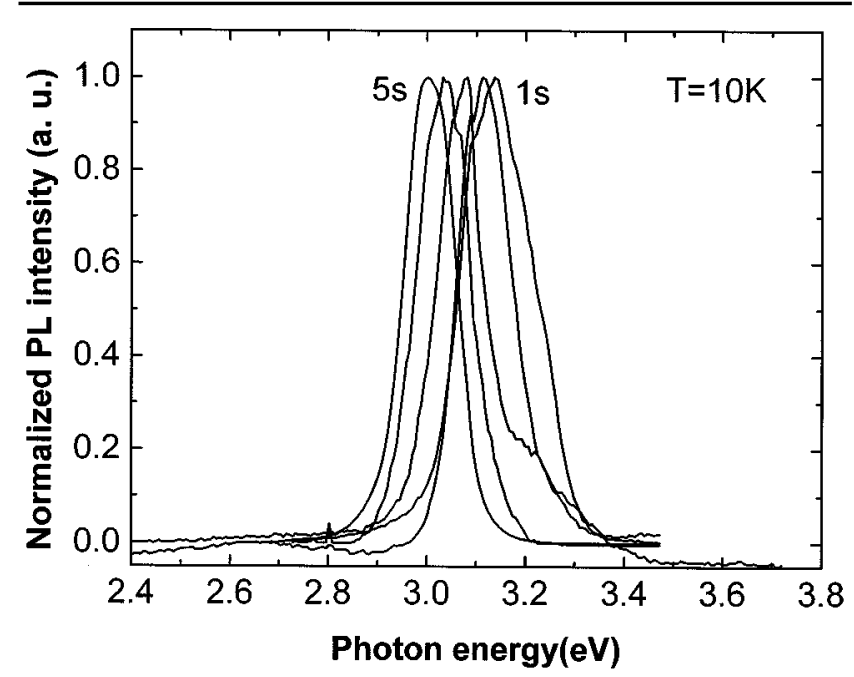

Fig. 2. From right to left, PL spectra for the $1 \mathrm{~s}, 2 \mathrm{~s}, 3 \mathrm{~s}, 4 \mathrm{~s}$, and $5 \mathrm{~s}$ samples taken at $\mathrm{T}=10 \mathrm{~K}$. The peak PL positions are at 3.14, 3.11, $3.08,3.04$, and $3.01 \mathrm{eV}$, respectively.

cal flow rates were $10 \mu \mathrm{mol} / \mathrm{min}, 30 \mu \mathrm{mol} / \mathrm{min}$, $30 \mu \mathrm{mol} / \mathrm{min}$, respectively. The growth pressure is kept at 760 torr, and during the growth $1500 \mathrm{sccm}$ purified $\mathrm{H}_{2}$ gas was fed into the growth chamber. The substrates were heated to $550^{\circ} \mathrm{C}$ in DES ambient for 40 min prior to sample growth. For all samples, the rough surfaces were created by growing $600 \mathrm{~A}$ of $\mathrm{ZnSe}$ at $400^{\circ} \mathrm{C}$, followed by growing $500 \AA$ of $\mathrm{ZnS}$ at $530^{\circ} \mathrm{C}$. Ten cycles of $\mathrm{ZnSe}$ and $\mathrm{ZnS}$ were then grown alternatively on this rough surface at $530^{\circ} \mathrm{C}$. In the alternation, the growth was interrupted for $10 \mathrm{~s}$. At this temperature the growth rates are calibrated to be $10 \AA /$ sec for $\mathrm{ZnSe}$ and $16 \AA ̊ 8 / \mathrm{sec}$ for $\mathrm{ZnS}$, respectively. The growth time for $\mathrm{ZnS}$ in each cycle is fixed at $40 \mathrm{sec}$ for all samples. The growth time for $\mathrm{ZnSe}$ in each cycle is also fixed for each sample but vary from 1 second to $10 \mathrm{sec}$ for different samples. We'll hereafter label the samples according to their ZnTe growth time in each cycle, i.e., $1 \mathrm{~s}$ sample, $2 \mathrm{~s}$ sample, etc. A schematic diagram of the sample structure is shown in Fig. 1.

Photoluminescence measurements on the samples were performed using a $\mathrm{He}-\mathrm{Cd}$ laser at an average

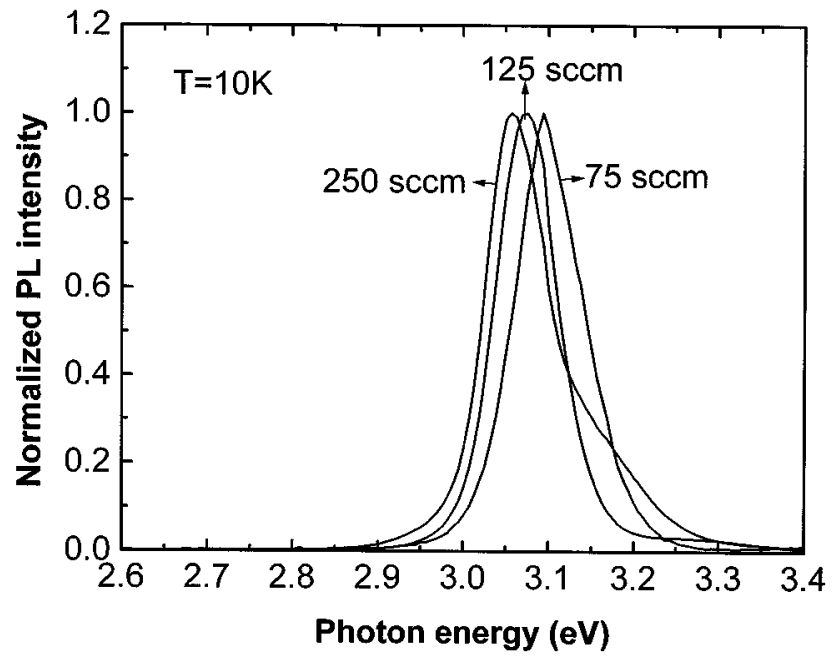

Fig. 3. PL spectra taken at different $D E S e$ flow rate. The flow rates are, from right to left, $75 \mathrm{sccm}, 125 \mathrm{sccm}$, and $250 \mathrm{sccm}$.

power of $50 \mathrm{~mW} / \mathrm{cm}^{2}$. A $0.85 \mathrm{~m}$ double monochromator, a photo-multiplier tube and standard lock-in technique were used to detect the signal. Figure 2 shows the PL emission spectra taken at $10 \mathrm{~K}$ for the $1 \mathrm{~s}$ to $5 \mathrm{~s}$ samples. It can be seen from this figure that the peak positions of the PL occur at $3.14 \mathrm{eV}$ for the $1 \mathrm{~s}$ sample, and decreases gradually with longer growth duration. For the 5s sample, the peak energy is at $3.01 \mathrm{eV}$. The PL emission energy for bulk ZnSe, at the same temperature, is known to occur at $2.82 \mathrm{eV}$. The amounts of blue shifts are between $190 \mathrm{meV}$ and $320 \mathrm{meV}$ for samples with different growth time. These large blue shifts are attributed to the effect of quantum confinement. The shorter the growth time, the smaller the quantum dot size. As the size decreases, stronger quantum confinement pushes up the subband energy in the quantum dots and result in a larger blue shift. In order to rule out the possibilities that the PL is from the $\mathrm{ZnS}$ layer, we have also performed the measurement with a sample with $\mathrm{ZnTe}$ growth time set at $0 \mathrm{~s}$. No PL could be observed for the sample. For the samples with growth duration time longer than $5 \mathrm{~s}$, no above bandedge emission was observed. The PL spectrum was dominated by below bandedge emission, and the intensity is 100 times smaller than the $1 \mathrm{~s}$ sample. This effect could be attributed the generation of defect as the size of the quantum dots become too large.

The effect of the flow rate on the size of the QDs was studied. Figure 3 depicts the PL spectra taken with growth time set to $1 \mathrm{~s}$ and the flow rate of the DESe set at $75 \mathrm{sccm}, 125 \mathrm{sccm}$, and $250 \mathrm{sccm}$, respectively. We can see from this figure that the smaller the flow rate, the larger the PL emission energy. This is because with the same growth time, the smaller the flow rates the lesser the available material, and the smaller the quantum dot. These results show that, in addition to the growth time, flow rate is another parameter that could be used to control the size of the QDs.

We had also studied the effect of coupling between layers on the PL properties of the quantum dots. 


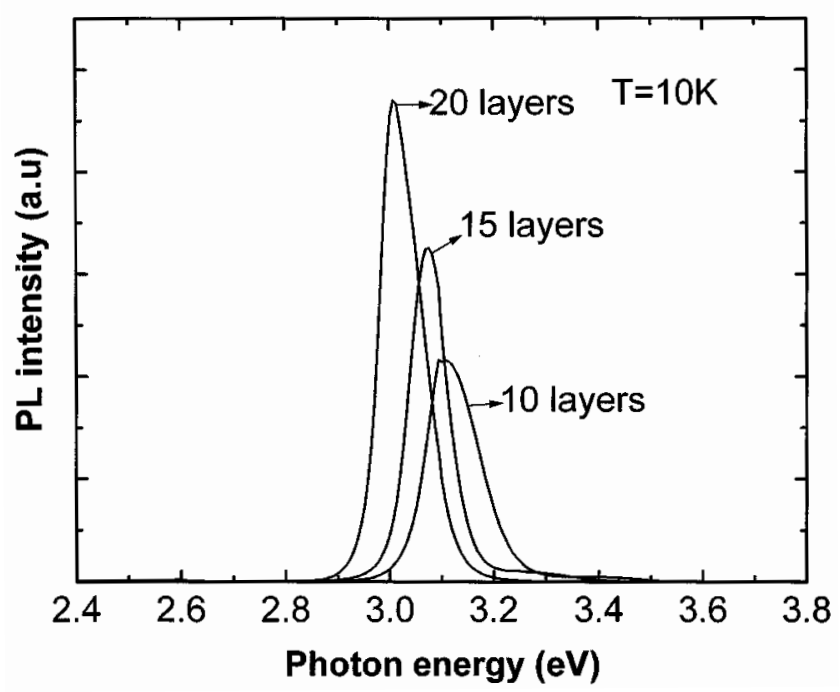

Fig. 4. PL spectra, from right to left, taken with 10, 15, and 20 layers of $\mathrm{ZnSe}$ quantum dots.

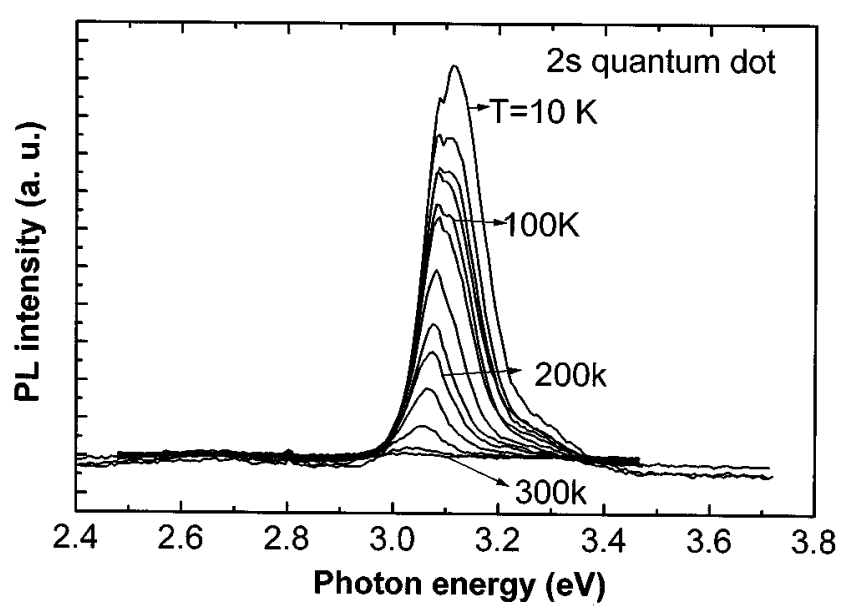

Fig. 5. Temperature-dependent PL spectra taken with the quantum dot growth time set at $2 \mathrm{~s}$. The temperatures, from top to bottom are: 10 $\mathrm{K}, 25 \mathrm{~K}, 50 \mathrm{~K}, 75 \mathrm{~K}, 100 \mathrm{~K}, 125 \mathrm{~K}, 150 \mathrm{~K}, 175 \mathrm{~K}, 200 \mathrm{~K}, 225 \mathrm{~K}, 250 \mathrm{~K}$, $275 \mathrm{~K}$, and $300 \mathrm{~K}$, respectively.

Figure 4 shows the PL spectra of samples grown under same condition but with 10,15, and 20 layers of quantum dots, respectively. We can see from this figure that PL intensity increases when there are more layers of quantum dots. In addition, we could also find that the peak PL energy shifts to lower energy and, at the same time, the full width at half maximum (FWHM) of the PL spectrum also decreases. The FWHM of the 10,15 , and 20 layers are $115 \mathrm{meV}$, $86 \mathrm{meV}$, and $84 \mathrm{meV}$, respectively. The results suggest that with more layers, the size distribution of the QD becomes more uniform and the dot size become larger. Our results are consistent with the theoretical prediction that modification of the surface potential caused by the underlying QDs will make the size distribution of the QDs more uniform when there are more layers of QDs. ${ }^{9}$

The temperature-dependent PL was studied and Fig. 5 depicts a typical spectrum taken with growth time set to $2 \mathrm{~s}$. Figure 6 summarizes the variation of

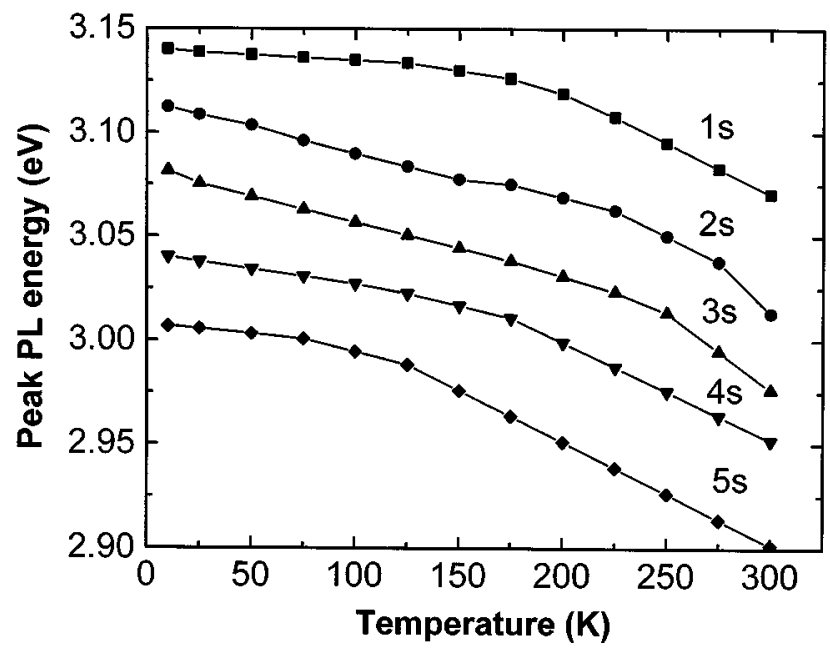

Fig. 6. The variation of the peak PL energy with temperature. The growth time of the quantum dots, from top to bottom are $1 \mathrm{~s}$ to $5 \mathrm{~s}$.

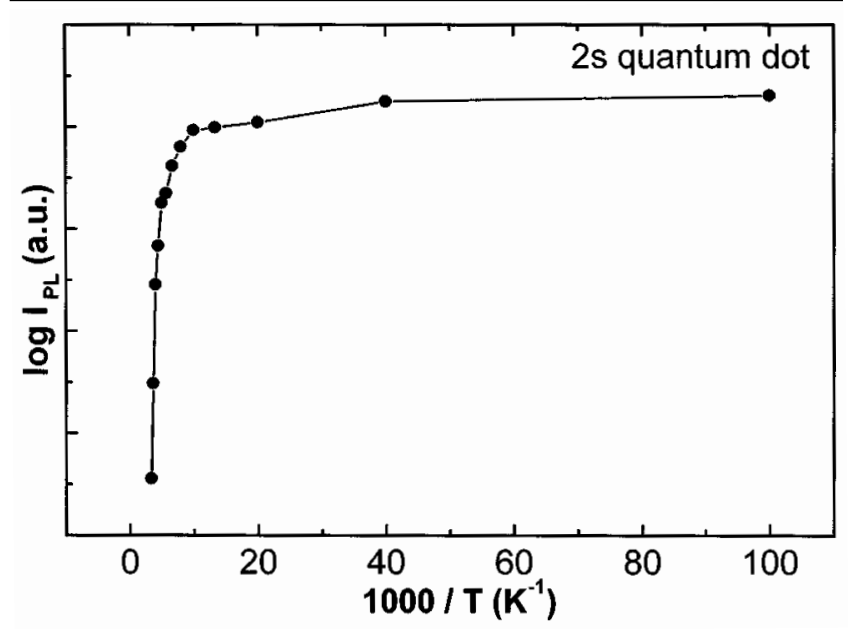

Fig. 7. Logarithm of the integrated PL intensity vs. inverse temperature for the $2 \mathrm{~s}$ sample.

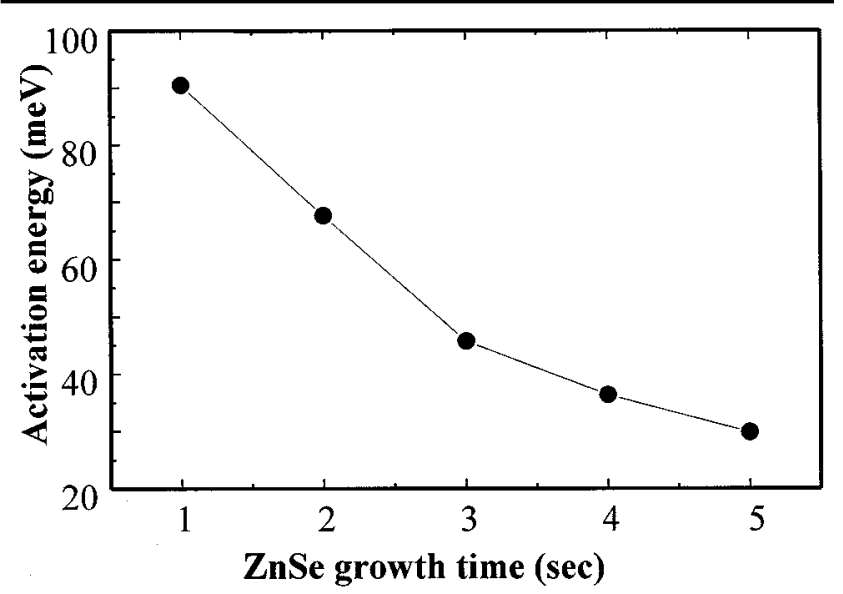

Fig. 8. The activation energy for quantum dots of growth time between $1 \mathrm{~s}$ and $5 \mathrm{~s}$.

the peak PL energy with temperature. For all the samples, independent of the growth time, the peak energy decreases with increasing temperature. This behavior can be attributed to the effect of lattice 
dilation and electron-phonon interaction. For bulk semiconductor, the bandgap shrinkage is known to follow the Varshni relation. ${ }^{10}$ We found that the relation is also valid for the semiconductor QDs.

The temperature dependence of the integrated PL intensity $\left(\mathrm{I}_{\mathrm{PL}}\right)$ could be expressed as: ${ }^{11}$

$$
\mathrm{I}_{\mathrm{PL}}(\mathrm{T})=\mathrm{I}_{0} /\left(1+\mathrm{A} \exp \left(-\mathrm{E}_{\mathrm{a}}\right) / \mathrm{kT}\right)
$$

Here $\mathrm{T}$ is temperature in Kelvin, $\mathrm{k}$ is Boltzmann constant, $\mathrm{I}_{0}$ and $\mathrm{A}$ are constants, and $\mathrm{E}_{\mathrm{a}}$ is the activation energy. By plotting $\log \left(\mathrm{I}_{\mathrm{PL}}\right)$ vs. $1 / \mathrm{T}$, the activation energy responsible for the quenching of PL could be obtained. A typical plot is shown in Fig. 7. The activation energy obtained by this method is usually considered as the total binding energy, which include the confinement energy and the exciton binding energy. The total binding energy, in general, was found to decrease with decreasing well width in a quantum well system. This is mainly because the narrow well width pushes up the subband energy and makes it easier for the electron to escape from the quantum wells. However, as are shown in Fig. 8, we found that the activation energies are $90 \mathrm{meV}, 68 \mathrm{meV}, 46 \mathrm{meV}$, $36 \mathrm{meV}$, and $30 \mathrm{meV}$ for the $1 \mathrm{~s}, 2 \mathrm{~s}, 3 \mathrm{~s}, 4 \mathrm{~s}$, and $5 \mathrm{~s}$ samples, respectively. The shorter the growth duration, the smaller the QD size, and the larger the activation energy. This is opposite to what was observed in conventional quantum well systems. This surprising result is believed to be due to the special band alignment of the $\mathrm{ZnS} / \mathrm{ZnSe}$ system.

Although the exact band alignment in the $\mathrm{ZnS} /$ ZnSe is still a subject of debate, it is generally believed that the band offset occurs mostly in the valence band..$^{12}$ There is little band offset in the conduction band (a type-II alignment is also a possibility). Since there is little (or no) confinement of the electron in $\mathrm{ZnSe}$, the energy required to quench PL is the energy required to break up exciton. After the exciton is broken apart, electron is free to diffuse into the barrier, and the PL is quenched. Therefore, it is the exciton binding energy, not the total binding energy that determines the quenching of the PL in the $\mathrm{ZnS} /$ ZnSe system. Wu and Nurmikko calculated the exciton binding energy in a quantum well with small valence band offset. ${ }^{13}$ They found that for a $50 \AA \mathrm{CdTe} /$ CdMnTe quantum well with zero valence band offset, the exciton binding energy is $22 \mathrm{meV}$. This is significantly larger than the exciton binding energy ( $14 \mathrm{meV}$ ) in bulk CdTe. Since our quantum dot has smaller size and is confined in all three directions, the exciton binding energy can be expected to be much larger than the bulk exciton binding energy in $\mathrm{ZnSe} .{ }^{14,15} \mathrm{We}$ thus conclude that the observed activation energy is the exciton binding energy in ZnSe quantum dot.

In conclusions, we have applied the flow-durationcontrolled method to grow ZnTe quantum dots embedded in $\mathrm{ZnS}$. We found that the shorter the growth time, the smaller the QDs, and the larger the blue shift. We showed that the flow rate could also be used to control the size of the quantum dots. The coupling between the quantum dot layers was found to have the effect of making the size distribution more uniform. The peak PL energy of the quantum dots is found to follow the Varshni rule. From the study of the quenching of PL it is found that the breaking up of the exciton is responsible for the quenching of PL at high temperature. The binding energy of exciton in the QDs is found to be substantially higher than the binding energy of exciton in bulk ZnzSe.

This work is supported by the National Science Council of the Republic of China.

\section{REFERENCES}

1. Y. Arakawa and H. Sakaki, Appl. Phys. Lett. 40, 939 (1982).

2. D. Bimberg, N.N. Ledentsov, M. Grundmann, N. Kirstaedter, O.G. Schmidt, M.H. Mao, V.M. Ustinov, A.Y. Egorov, A.E. Zhukov, P.S. Kopev, Z.I. Alferov, S.S. Ruvimov, U. Gosele, and J. Heydenreich, Jpn. J. Appl. Phys., part I 35, 1311 (1996).

3. D.J. Eaglesham and M. Cerullo, Phys. Rev. Lett. 64, 1943 (1990).

4. M. Longo, N. Lovergine, A.M. Mancini, A. Passaseo, G. Leo, M. Mazzer, M. Berti, and A.V. Drigo,Appl. Phys. Lett. 72, 359 (1998).

5. B.P. Zhang, W.X. Wang, T. Yasuda, Y. Segawa, K. Edmatsu, and T. Itoh, Appl. Phys. Lett. 71, 3370 (1997)

6. Y.D. Dai, J.C. Fan, Y.F. Chen, R.M. Lin, S.C. Lee, and H.H. Lin, J. Appl. Phys. 82, 4489 (1997).

7. D.J. Engleshan and M. Cerullo, Phys. Rev. Lett. 64, 1943 (1990).

8. M.C. Harris Liao, Y.H. Chang, Y.F. Chen, J.H. Hsu, J.M. Lin, and W.C. Chou, Appl. Phys. Lett. 70, 2256 (1997).

9. J. Tersoff, G. Teichert, and M.G. Lagally, Phys. Rev. Lett. 76, 1675 (1996).

10. Y.P. Varshni, Physica 34, 149 (1967).

11. J.D. Lambkin, D.J. Dunstan, K.P. Homewood, and L.K. Howard, Appl. Phys. Lett. 57, 1986 (1990).

12. R. Cingolani, M. Lomascolo, N. Lovergine, M. Dabbicco, M. Ferrara, and I. Suemune, Appl. Phys. Rev. 64, 2439 (1994).

13. J.W. Wu and A.V. Nurmikko, Phys. Rev. B 38, 1504 (1988)

14. M. Boero, J.M. Rorison, G. Duggan, and J.C. Inkson, Surf. Sci. 377-379, 371 (1997).

15. J.L. Marin, R. Riera, and S.A. Cruz,J. Phys: Condens. Matter 10, 1349 (1998). 